\title{
KHÁM PHÁ VAI TRÒ CỦA CHÂT LƯợNG CẢM NHẬN VÀ CHI PHÍ CHUYỂN ĐỔI LÊN LÒNG TRUNG THÀNH THƯƠNG HIẸU CỦA KHÁCH HÀNG SỦ DỤNG MẠNG DI ĐỘNG Ở VIẸT NAM
}

\section{EXPLORING THE EFFECT OF QUALITY PERCEIVED AND CUSTOMER SWITCHING COSTS ON MOBILE PHONE CUSTOMER'S BRAND LOYALTY IN VIETNAM}

Ngày nhận bài: 23/02/2019 Ngày chấp nhận đăng: 18/3/2019 ～Ngày đăng: 05/10/2019

Nguyễn Thị Hồng Nguyệt ${ }^{1}$

\section{Tóm tắt}

Nghiên cứu này xem xét các thành phần của chi phí chuyển đổi và sự ảnh hưởng của nó đối với lòng trung thành thương hiệu. Bên cạnh đó, yếu tố chất lượng cảm nhận cũng được xem xét trong mối quan hệ với chi phí phí chuyển đổi và lòng trung thành thương hiệu. Mẫu nghiên cứu là 300 khách hàng được phỏng vấn trực tiếp bằng bảng câu hỏi và phương pháp mô hình cấu trúc tuyến tính SEM được sử dụng để phân tích dữ liệu. Kết quả nghiên cứu cho thấy chi phí chuyển đổi không có ảnh hưởng đến lòng trung thành thương hiệu, nhưng chất lượng cảm nhận là yếu tố quan trọng có tác động tích cực đến chi phí chuyển đổi và lòng trung thành thương hiệu.

Tì khóa: Chi phí chuyển đổi, chất lượng cảm nhận, lòng trung thành thương hiệu.

\begin{abstract}
This study examines the components of customer switching costs and its influence on brand loyalty. Besides, perceived quality is also been considered in the relationship between customer switching costs and brand loyalty. A sample of 300 consumers was surveyed to test the theoretical model. Structural equation modeling (SEM) was used to analyze the data. The research result confirms that customer switching costs had not effect on brand loyalty, and perceived quality affected on customer switching costs and brand loyalty.
\end{abstract}

Key words: Customer switching costs, perceived quality, and brand loyalty.

\section{Giới thiệu}

Lòng trung thành thương hiệu là một khái niệm quan trọng được các nhà nghiên cứu và quản trị thực tiễn đặc biệt quan tâm trong những năm gần đây. Trong lĩnh vực marketing lòng trung thành thương hiệu không chỉ được xem biến số trọng tâm của giá trị thương hiệu
(Lý thuyết thương hiệu và xây dựng thương hiệu; vd,. Aaker (1996), Yoo \& Donthu (1997), Nguyễn Đình Thọ và Nguyễn Thị Mai Trang (2011),...) mà còn được khẳng định là biến kết quả của quá trình xây dựng mối quan hệ có chất lượng lâu bền với khách hàng (theo Lý thuyết marketing mối quan hệ; vd,. De Wulf và cộng

\footnotetext{
${ }^{1}$ Trường Đại học Tài chính - Marketing
} 
sự (2001), Hennig-Thurau và cộng sự (2002), Liu và cộng sự (2011),...).

Có rất nhiều nghiên cứu xem xét các yếu tố tiền đề của lòng trung thành thương hiệu trong nhiều lĩnh vực, điều kiện khác nhau như hình ảnh doanh nghiệp và hình ảnh thương hiệu (Chen và Myagmarsuren, 2011), chất lượng dịch vụ, sự thân mật (Liu và cộng sự, 2011),... Tuy nhiên, rất ít nghiên cứu tìm hiểu mối quan hệ giữa yếu tố chi phí chuyển đổi và lòng trung thành thương hiệu đặc biệt là tại thị trường Việt Nam - một thị trường tiêu dùng tiềm năng có mức tăng trưởng cao nhất ở khu vực ASEAN (Global Insights, Bain Analysis, trích từ Hoa Vinh, 2013). Hơn nữa, lĩnh vực cung cấp dịch vụ mạng viễn thông di động ở Việt Nam cũng được xem là một ngành tiềm năng và mang tính cạnh tranh cao khi nhà nước Việt Nam phá thế độc quyền chuyển sang nền kinh tế thị trường. Vì vậy, nghiên cứu này sẽ tìm hiểu mối quan hệ giữa chi phí chuyển đổi và lòng trung thành thương hiệu trong lĩnh vực tiêu dùng dịch vụ mạng viễn thông di động tại Việt Nam.

\section{Co sở lý thuyết và các giả thuyết nghiên cứu}

Vai trò của chất lượng cảm nhận và chi phí chuyển đổi đối với lòng trung thành thương hiệu được các nhà nghiên cứu thực hiện trong những nghiên cứu độc lập. Chất lượng cảm nhận được khẳng định là nhân tố quan trọng có tác động lên lòng trung thành thương hiệu trong nghiên cứu của Nguyễn Đình Thọ và Nguyễn Thị Mai Trang (2011). Trong khi, chi phí chuyển đổi có ảnh hưởng tích cực lên lòng trung thành thương hiệu được tìm thấy trong nghiên cứu của Gremler và cộng sự (1996), Jones và cộng sự (2002), Arasil và cộng sự (2005), Willys (2018). Gatignon và Robertson (1992) và Klemperer (1995) đã cho thấy có mối quan hệ giữa chất lượng cảm nhận và chi phí chuyển đổi; trong đó chất lượng cảm nhận là nhân tố tiền đề có tác động đến chi phí chuyển đổi. Trên cơ sở của các nghiên trước, các khái niệm và giả thuyết nghiên cứu sẽ được trình bày chi tiết ở phần tiếp theo.

\subsection{Nhũng khái niệm nghiên cứu}

\section{Chất lượng cảm nhận}

Theo Dawa (1999), chất lượng cảm nhận là những ý kiến, đánh giá tổng quan của khách hàng về sự vượt trội, tuyệt hảo hay ở những độ khác nhau nào đó của sản phẩm. Vì vậy, chất lượng cảm nhận là những nhận xét, cảm nhận mang tính chủ quan của người tiêu dùng về chất lượng sản phẩm. Nó sẽ phân biệt và ít khi trùng lắp với chất lượng thật sự của sản phẩm vì khách hàng thường không phải là những chuyên gia và nhận xét của họ có phần ảnh hưởng từ hình ảnh của thương hiệu, từ cảm tình đối với thương hiệu, từ những thông điệp quảng cáo hoặc từ những lời tác động, tán dương của những người thân quen. Do đó, người tiêu dùng khác nhau sẽ có chất lượng cảm nhận khác nhau.

\section{Chi phí chuyển đổi}

Chi phí chuyển đổi là chi phí liên quan mà người mua phải đối mặt khi chuyển đổi từ một nhà cung cấp sản phẩm này sang một nhà cung cấp sản phẩm khác. Có rất nhiều quan điểm về chi phí chuyển đổi và các thành phần của nó, và hiện nay chưa có sự thống nhất giữa các nhà nghiên cứu. Trong phạm vi nghiên cứu này, chi phí chuyển đổi sẽ được xem xét theo quan điểm của Brunham và cộng sự (2003). Brunham và cộng sự (2003) cho rằng chi phí chuyển đổi bao gồm ba thành tố: chi phí liên quan đến thủ tục chuyển đổi, chi phí liên quan đến tài chính và chi phí liên quan đến mối quan hệ. Trong đó, chi phí liên quan đến thủ tục bao gồm chi phí về thời gian, chi phí học tập, trải nghiệm và đánh giá giá trị dịch vụ, sản phẩm của nhà cung cấp mới; Chi phí chuyển đồi tài chính liên quan đến các nguồn lực tổn thất tài chính của người mua 
bao gồm tổn thất tiền tệ (mua dịch vụ, sản phẩm mới của nhà cung cấp khác) và chi phí tổn thất lợi ích (các điểm tích lũy, chương trình khách hàng thân thiết,...); và chi phí chuyển đổi quan hệ là chi phí liên quan đến sự khó chịu về cảm xúc hoặc tâm lý do mất nhận dạng và phá vỡ mối quan hệ bao gồm chi phí mất mối quan hệ cá nhân và chi phí mất mối quan hệ thương hiệu (Brunham và cộng sự, 2003).

\section{Lòng trung thành thuoong hiệu}

Lòng trung thành thương hiệu là một trong những khái niệm quan trọng của lý thuyết thương hiệu, được các nhà nghiên cứu và quản trị thực tiễn chú ý nhiều trong những năm gần đây. Lòng trung thành là khi khách hàng có xu hướng mua nhiều sản phẩm của một thương hiệu nào đó và lặp lại hành vi này (Chaudhuri, 1999) hay lòng trung thành thể hiện thái độ của khách hàng, nếu khách hàng tin tưởng và có ấn tượng tốt về một thương hiệu thì sẽ ưu tiên tìm mua sản phẩm của thương hiệu đó (Yoo \& đtg, 2000).

\subsection{Giả thuyết nghiên cứu}

Chất lượng cảm nhận và chi phí chuyển đổi và lòng trung thành thwơng hiệu

Chất lượng cảm nhận là một trong những nhân tố quan trọng để khách hàng đánh giá và quyết định duy trì nhà cung cấp sản phẩm, dịch vụ hay thay đổi sang nhà cung cấp khác; và vì vậy ảnh hưởng đến lòng trung thành của khách hàng đối với thương hiệu đang sử dụng. Thật vậy, Gatignon và Robertson (1992) và Klemperer (1995) xem xét đặc trưng của sản phẩm như yếu tố tạo ra chi phí chuyển đổi. Đối với sản phẩm càng phức tạp khó sử dụng thì rủi ro càng lớn, chi phí chuyển đổi càng cao (Holak \& Lehmann, 1990); và khách hàng có khuynh hướng duy trì mối quan hệ với thương hiệu hiện tại hơn (Sheth \& Parvatiyar, 1995). Vì vậy, các giả thuyết được đưa ra là:

H1: Chất luợng cảm nhận có ảnh hưởng tích cực đến chi phí chuyển đổi
H2: Chất luợng cảm nhận có ảnh hưởng tích cưc đến lòng trung thành thuoong hiệu.

Chi phí chuyển đổi và lòng trung thành thuoong hiệu

Nghiên cứu của Gremler và cộng sự (1996) đã chứng minh chi phí chuyển đổi làm tăng lòng trung thành của khách hàng; điều này cho thấy chính những lo ngại về chi phí phát sinh như tìm hiểu, học tập, làm quen với cái mới, mua thiết bị, công nghệ, sản phẩm mới hay phải từ bỏ những lợi ích đã tích lũy trong quá trình chuyển đổi nhà cung cấp khác khiến khách hàng có khuynh hướng duy trì mối quan hệ với nhà cung cấp dịch vụ, sản phẩm hiện có. Trong nghiên cứu của mình Jones và cộng sự (2002), Arasil và cộng sự (2005), Willys (2018),... cũng khẳng định chi phí chuyển đổi có ảnh hưởng đến lòng trung thành của khách hàng. Vì vậy, giả thuyết được đưa ra là:

H3: Chi phí chuyển đổi có ảnh hưởng tích cực đến lòng trung thành thuoong hiệu.

\subsection{Mô hình lý thuyết}

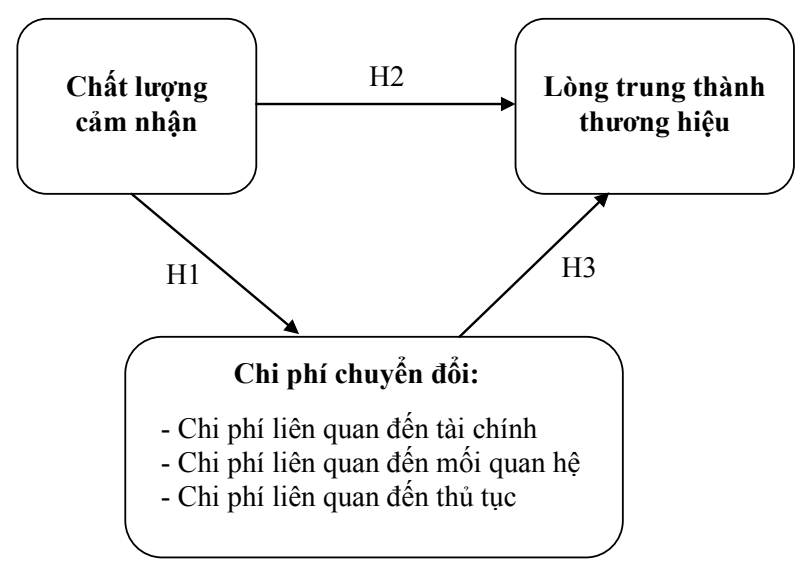

Hình 1. Mô hình nghiên cứu lý thuyết

\section{Phương pháp nghiên cứu}

Nghiên cứu được tiến hành thông qua hai bước: (1) Nghiên cứu định tính; (2) Nghiên cứu định lượng. Nghiên cứu định tính được thực hiện bằng phương pháp phỏng vấn sâu với đối tượng là khách hàng có sử dụng mạng 
viễn thông di động. Nghiên cứu định tính nhằm khám phá nhận thức khách hàng về thang đo sẵn có đồng thời điều chỉnh cho phù hợp với điều kiện nghiên cứu.

Nghiên cứu định lượng sử dụng phương pháp lấy mẫu thuận tiện được thực hiện bằng cách phỏng vấn trực tiếp thông qua bảng câu hỏi đối với 300 người sử dụng mạng điện thoại di động. Trước tiên, dữ liệu sẽ được kiểm hệ số tin cậy Cronbach's alpha để loại bỏ những thang đo không đạt độ tin cậy. Kế đến, phân tích nhân tố khám phá (EFA) được áp dụng với chiến lược phân tích tách nhóm; có nghĩa là các khái niệm đa hướng hay còn gọi là khái niệm bậc hai sẽ được xử lý riêng và khái niệm bậc một sẽ được xử lý riêng. Tiếp theo, phân tích nhân tố khẳng định (CFA) được tiến hành với cách thức thực hiện cho khái niệm đa hướng trước, sau đó tiến hành thực hiện CFA cho mô hình tới hạn. Cuối cùng, mô hình cấu trúc tuyến tính (SEM) được ứng dụng để kiểm định giả thuyết và mô hình nghiên cứu. Các phân tích được thực hiện dưới sự hỗ trợ của phần mềm SPSS và $A M O S$.

\section{Kết quả nghiên cứu}

\section{1. Đặc điểm mẫu}

Kết quả khảo sát cho thấy tỷ lệ người dùng dịch vụ mạng điện thoại di động chủ yếu của Viettel, Mobifone và Vinaphone (chiếm 96.3\%). Trong đó tỷ lệ khách hàng trong mẫu điều tra sử dụng dịch vụ của nhà cung cấp Viettel chiếm tỷ lệ cao nhất (44.3\%), kế đến là Mobifone và Vinaphone lần lượt chiếm tỷ lệ $30 \%$ và $22 \%$. Con số cụ thể được thể hiện qua bảng 4.1.

\section{Bảng 1. Tỷ lệ người dùng dịch vụ mạng điện thoại di động của các nhà cung cấp trong mẫu khảo sát}

\begin{tabular}{|l|c|c|c|c|}
\hline \multicolumn{1}{|c|}{ Thương hiệu } & Tần suất & Phần trăm & Phần trăm hộp lệ & Phần trăm tích lũy \\
\hline Mobifone & 90 & 30,0 & 30,0 & 30,0 \\
\hline Vinaphone & 66 & 22,0 & 22,0 & 52,0 \\
\hline Viettel & 133 & 44,3 & 44,3 & 96,3 \\
\hline Vietnam mobile & 10 & 3,3 & 3,3 & 99,7 \\
\hline Khác & 1 & 0,3 & 0,3 & 0 \\
\hline Tổng cộng & 300 & 100,0 & 100,0 & 100,0 \\
\hline
\end{tabular}

Nguồn: Tù kết quả khảo sát của nghiên cưu

Người tiêu dùng trong lĩnh vực dịch vụ mạng điện thoại di động phần lớn ở độ tuổi từ 30 trở xuống (chiếm 68,3\%: từ 18 đến 22 tuổi chiếm $22 \%$ và từ $23-30$ tuổi chiếm $46,3 \%$ ); hầu hết có thu nhập từ 10 triệu trở xuống và nghề nghiệp chính là công nhân, viên chức. 
Bảng 2. Đặc điểm mẫu nghiên cứu

\begin{tabular}{|c|c|c|c|}
\hline \multirow{2}{*}{\multicolumn{2}{|c|}{ Đặc điểm mẫu }} & \multicolumn{2}{|c|}{ Thị trường cung cấp mạng di động } \\
\hline & & \multirow{2}{*}{$\begin{array}{c}\text { Số lượng } \\
140\end{array}$} & \multirow{2}{*}{$\begin{array}{c}\text { Tỷ lệ (\%) } \\
46,7\end{array}$} \\
\hline & Nam & & \\
\hline Giới tính & Nữ & 160 & 53,3 \\
\hline \multirow{6}{*}{ Thò̀i gian sử dụng } & Dưới 1 năm & 6 & 2,0 \\
\hline & Từ $1-<2$ năm & 14 & 4,7 \\
\hline & Từ $2-<3$ năm & 34 & 11,3 \\
\hline & Từ $3-<4$ năm & 50 & 16,7 \\
\hline & Từ $4-<5$ năm & 78 & 26,0 \\
\hline & Trên 5 năm & 18 & 39,3 \\
\hline \multirow{4}{*}{ Độ tuổi } & Từ 18 đến 22 & 66 & 22,0 \\
\hline & Từ 23 đến 30 & 139 & 46,3 \\
\hline & Từ 30 đến 40 & 58 & 19,3 \\
\hline & Trên 40 & 37 & 12,3 \\
\hline \multirow{4}{*}{$\begin{array}{l}\text { Thu nhập } \\
\text { (Triệu) }\end{array}$} & Dưới 5 triệu & 125 & 41,7 \\
\hline & Từ $5-<10$ & 114 & 38,0 \\
\hline & Từ $10-<20$ & 41 & 13,7 \\
\hline & Trên 20 & 20 & 6,7 \\
\hline \multirow{5}{*}{ Công việc } & Chưa đi làm & 29 & 9,7 \\
\hline & Nhân viên & 152 & 50,7 \\
\hline & Nhà quản lý & 31 & 10,3 \\
\hline & Công, viên chức & 37 & 12,3 \\
\hline & Khác & 51 & 17,0 \\
\hline
\end{tabular}

\subsection{Kiểm định thang đo}

Các thang đo được đánh giá độ tin cậy Cronbach's alpha và phân tích nhân tố khám phá EFA. Kết quả ban đầu cho thấy các thang đo đều đạt độ tin cậy và giá trị phân biệt. Tiếp đến, kết quả phân tích nhân tố khẳng định (CFA) cho thấy các thang đo đo lường các khái niệm nghiên cứu đều đạt giá trị.

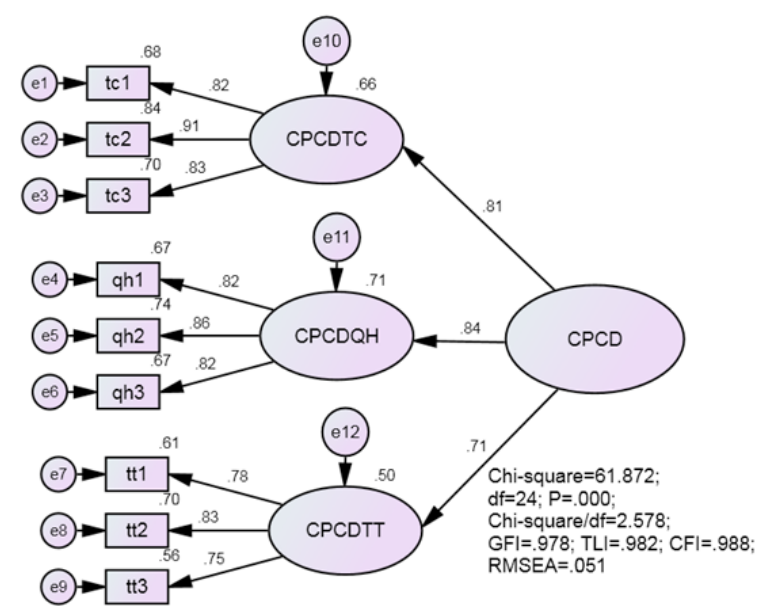

Hình 2. Kết quả CFA thang đo chi phí chuyển đổi 


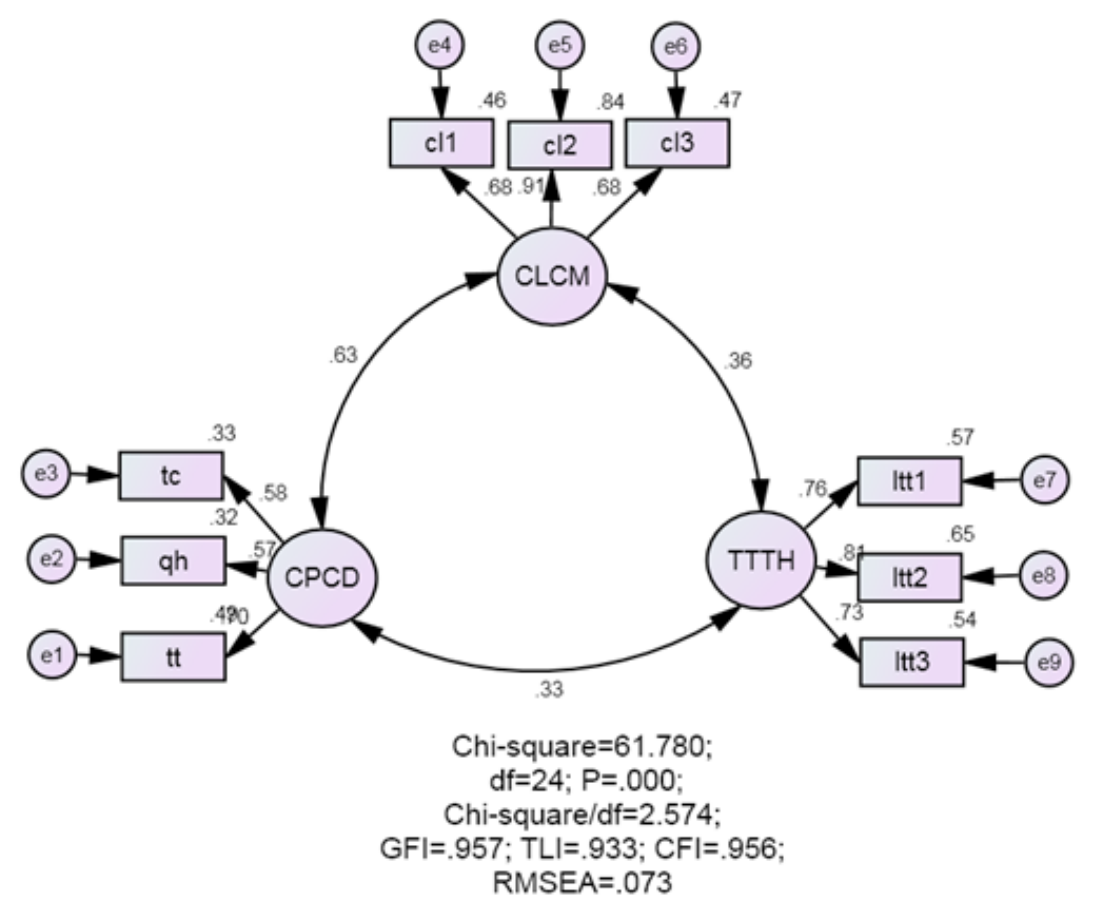

Hình 3. Kết quả phân tích CFA của mô hình tới hạn

Nguồn: Tù kết quả khảo sát của nghiên cưu

Bảng 3. Bảng tóm tắt kết quả kiểm định thang đo

\begin{tabular}{|l|c|c|c|c|c|}
\hline \multicolumn{1}{|c|}{$\begin{array}{c}\text { Các khái niệm } \\
\text { nghiên cứu }\end{array}$} & $\begin{array}{c}\text { Ký } \\
\text { hiệu }\end{array}$ & $\begin{array}{c}\text { Cronbach } \\
\text { alpha }\end{array}$ & $\begin{array}{c}\text { Độ tin cậy } \\
\text { tồng hộp }\end{array}$ & $\begin{array}{c}\text { Phương } \\
\text { sai trích }\end{array}$ & $\begin{array}{c}\text { Hệ số tải nhân } \\
\text { tố bình quân }\end{array}$ \\
\hline Chất lượng cảm nhận & CLCN & 0.787 & 0.808 & 0.588 & 0.759 \\
\hline $\begin{array}{l}\text { Chi phí chuyển đổi liên quan đến } \\
\text { tài chính }\end{array}$ & tc & 0.758 & 0.893 & 0.736 & 0.857 \\
\hline $\begin{array}{l}\text { Chi phí chuyển đổi liên quan đến } \\
\text { quan hệ }\end{array}$ & qh & 0.841 & 0.869 & 0.689 & 0.829 \\
\hline $\begin{array}{l}\text { Chi phí chuyển đổi liên quan đến } \\
\text { thủ tục }\end{array}$ & tt & 0.644 & 0.934 & 0.622 & 0.788 \\
\hline Chi phí chuyển đổi & CPCD & & 0.648 & 0.483 & 0.616 \\
\hline Lòng trung thành thương hiệu & TTTH & 0.807 & 0.809 & 0.586 & 0.765 \\
\hline
\end{tabular}

Nguồn: Tù kết quả khảo sát của nghiên cưu

\footnotetext{
4.3. Kiểm định mô hình lý thuyết và các giả thuyết

Mô hình hóa cấu trúc tuyến tính (SEM) được sử dụng để kiểm định mô hình lý thuyết và các giả thuyết. Phân tích SEM cho thấy mô
}

hình lý thuyết phù hợp với dữ liệu thị trường với chỉ số Chi-square $=61.780(p=000)$; Chisquare/ $\mathrm{df}=2.574$, GFI $=0.957$; TLI $=0.933$; $\mathrm{CFI}=0.956 ; \mathrm{RMSEA}=0.73$. 


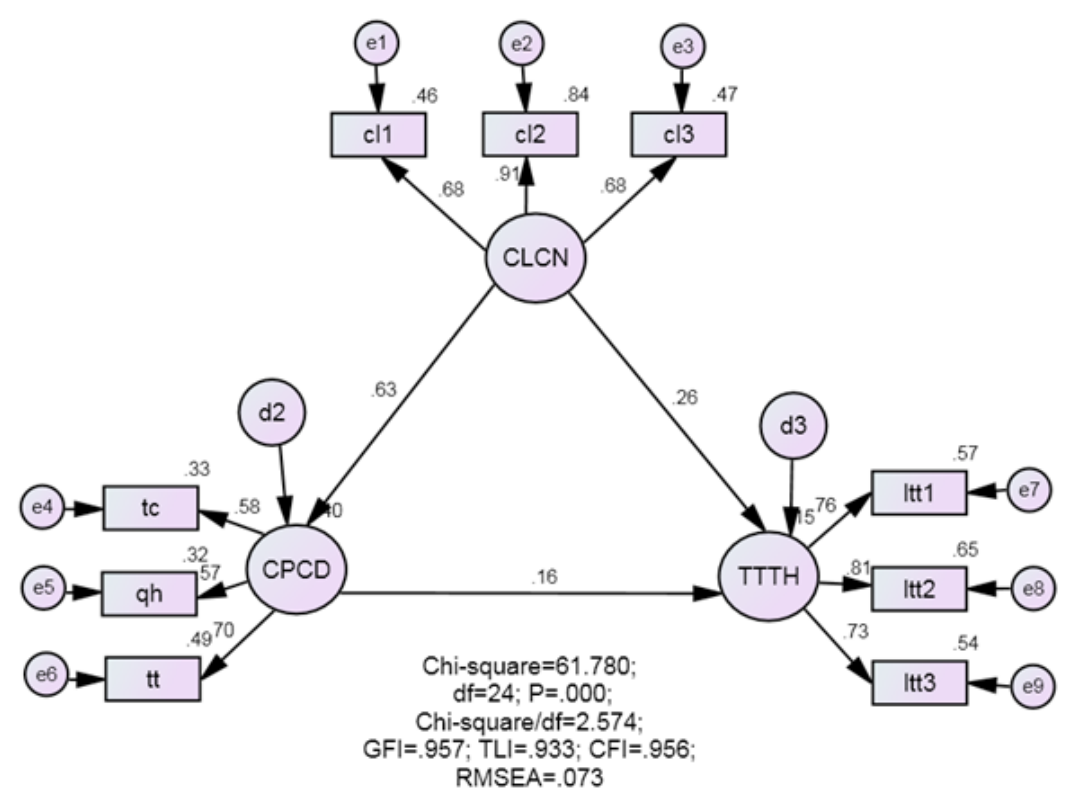

Hình 4. Kết quả SEM (chuẩn hóa) của mô hình nghiên cứu

Nguồn: Tù kết quả khảo sát của nghiên cưu

Các mối tương quan được giả thuyết trong mô hình nghiên cứu đều được kiểm định bằng mô hình cấu trúc tuyến tính SEM. Kết quả phân tích cho thấy chất lượng cảm nhận có ảnh hưởng tích cực đến chi phí chuyển đổi và lòng trung thành thương hiệu; điều này có nghĩa là giả thuyết $\mathrm{H} 1$ và $\mathrm{H} 2$ được chấp nhận. Kết quả cũng cho thấy giả thuyết $\mathrm{H} 3$ không được chấp nhận vì có giá trị $\mathrm{p}>0.05$. Vì vậy, có thể khẳng định chi phí chuyển đổi không có ảnh hưởng đến lòng trung thành thương hiệu.

Bảng 4. Hệ số hồi quy (chưa chuẩn hóa) của mô hình lý thuyết

\begin{tabular}{|c|c|c|c|c|}
\hline Mối quan hệ & Ước lượng & S.E. & C.R & P \\
\hline $\mathrm{CLCN} \rightarrow \mathrm{CPCD}$ & 1.530 & .238 & 6.428 & $* * *$ \\
\hline $\mathrm{CLCN} \rightarrow \mathrm{TTTH}$ & .310 & .119 & 2.604 & .009 \\
\hline $\mathrm{CPCD} \rightarrow \mathrm{TTTH}$ & .076 & .054 & 1.411 & .158 \\
\hline
\end{tabular}

Nguồn: Tù kết quả khảo sát của nghiên cưu

Bảng 5. Kết quả kiểm định các giả thuyết nghiên cứu

\begin{tabular}{|c|l|c|c|}
\hline Giả thuyết & \multicolumn{1}{|c|}{ Mối quan hệ giữa các khái niệm } & p & Kết luận \\
\hline H1 & Chất lượng cảm nhận $\rightarrow$ chi phí chuyển đổi & $* * *$ & Chấp nhận \\
\hline H2 & Chất lượng cảm nhận $\rightarrow$ lòng trung thành & 0.009 & Chấp nhận \\
\hline H3 & Chi phí chuyển đổi $\rightarrow$ lòng trung thành & 0.158 & Không nhận \\
\hline
\end{tabular}

Nguồn: Tù kết quả khảo sát của nghiên cưu 


\section{Kết luận và hàm ý quản trị}

Kết quả cho thấy mô hình lý thuyết phù hợp với dữ liệu thị trường. Chất lượng cảm nhận đóng vai trò quan trọng vì có ảnh hưởng tích cực đến chi phí chuyển đổi và lòng trung thành thương hiệu tương tự như những nghiên cứu trước. Tuy nhiên, không giống như những nghiên cứu trước (Vd., Jones và cộng sự, 2002; Arasil và cộng sự, 2005; Willys, 2018), dữ liệu trong nghiên cứu này cho thấy chi phí chuyển đổi là yếu tố không có ảnh hưởng lên lòng trung thành thương hiệu về ý nghĩa thống kê. Điều này đã phản ánh được tình hình thực tế là chất lượng cảm nhận của mỗi thương hiệu được cảm nhận là khác nhau nhưng giá cho chi phí chuyển đổi sang thương hiệu khác không là vấn đề với khách hàng vì chi phí mua sim của nhà cung cấp dịch vụ di động mới là không cao, thủ tục để mua thêm dịch vụ của các nhà cung cấp mạng di động là không phức tạp, thậm chí những gói cước, dịch vụ khuyến mãi khi hòa sóng di động của các thương hiệu là vô cùng hấp dẫn (đổi mạng vẫn giữ số liên lạc cũ, chọn số đẹp không thu thêm phí, gói cước rẻ nhất cho dung lượng lớn nhất, hay gọi cùng mạng sẽ không tính tiền cước, ... ). Hơn nữa, sự cạnh tranh gay gắt giữa các nhà sản xuất đã cho ra những thiết bị (mobile phone) có thể cùng lúc có thể sử dụng nhiều thương hiệu của các nhà cung cấp dịch mạng di động với chi phí thấp đã giúp cho người tiêu dùng dễ dàng sở hữu những chiếc điện thoại có sử dụng nhiều thương hiệu của các nhà cung cấp dịch vụ di động nhiều hơn. Chính việc mỗi người khách hàng đều có khả năng sử dụng nhiều nhà cung cấp dịch vụ khác nhau đã tạo nên sự kết nối với các mối quan hệ của mình với chi phí thấp hơn.

Tóm lại, với kết quả nghiên cứu này nhà quản trị trong lĩnh vực cung cấp mạng viễn thông di động cho thị trường hàng tiêu dùng có thể tập trung các nhóm giải pháp nhằm nâng cao chất lượng cảm nhận (đường truyền tín hiệu tốt, nhanh không bị tắc nghẽn, chi phí phù hợp, các dịch vụ chăm sóc khách hàng/ khách hàng thân thiết luôn hoạt động tốt,...) nhằm làm giảm thiểu chi phí chuyển đổi và tăng lòng trung thành thương hiệu của khách hàng.

\section{Tài liệu tham khảo}

Aaker, D. A, 1996. Measuring brand equity across products and markets. California Management Review, 38 (3), 102-120.

Arasil, O., Ozer, G. \& Aydin, S., 2005. Customer loyalty and the effect of switching cost as a moderator variable. Marketing Intelligence \& Planning, 23 (1): 89-103.

Burnham, T. A., Frels J. K., \& Mahajan., 2003. Consumer Switching Costs: A Typology, Antecedents, and Consequences. Journal of the Academy of Marketing Science, 31, 109-126.

Callaghan, M.; J. Mcphail \& O. H. M. Yau (1995), Dimensions of a Relationship Marketing Orientation: An Empirical Exposition, Proceedings of the Seventh Biannual Word Marketing Congress, 7 (2), pp. $10-65$.

Chaudhuri, A., (1999), Does Brand Loyalty Mediate Brand Equity Outcomes?, Journal of Marketing Theory and Practice, Spring 99, 136 - 146.

Chen, C. \& Myagmarsuren, O. (2011), Brand Equity, relationship quality, relationship value, and customer loyalty: Evidence from the telecommunications services, Total Quality Management, iFrist Article, $1-18$. 
Dawa, N. (1999), Perceived quality. In P.E. Earl and S.Kemp (Eds) The Elgar Companion to Consumer Research and Economic Psychology, Northampton, MA: Edward Elgar.

De Wulf, K., Odekerken-Schroder, G. \& Iacobucci, D., 2001. Investments in Consumer Relationships: A Cross-Country and Cross-Industry Exploration. Journal of Marketing, 65 (4), pp.33-50.

Gatignon, Hubert and Thomas S. Robertson., 1992. The Handbook of Consumer Behavior. Eds. Thomas S. Robertson and Harold H. Kassarjian, Englewood Cliffs, NJ: Prentice Hall.

Gremler, D. D. \& Brown, S.W., 1996. Service Loyalty: Its Nature, Importance, and Implications. pp. 171-180. ISQA C/o Business research Institue.

Hennig - Thurau T., Gwinner, K. P., Gremler, D.D., 2002. Understanding relationship Marketing outcomes: An Integration of Relational Benefits and Relationship Quality. Journal of Service Resaerch, 4 (3), 230-47.

Hoa Vinh, 2013. Việt Nam, "ngôi sao" mới ngành hàng tiêu dùng. <http://vneconomy.vn/thitruong/viet-nam-ngoi-sao-moi-nganh-hang-tieu-dung-20130730102544430.htm> [Ngày truy cập, 16 tháng 02 năm 2019].

Holak, Susan L. \& Donald R. Lehmann., 1990. Purchase Intentions and the Dimensions of Innovation: An Explorarory Model. Journal of Product Innovation Management. 7:59-73.

Jones, M.A., Mothersbaugh, D.L., Beatty, S.E., 2002. Why customer stay: measuring the underlying dimensions of services switching costs and managing their differential strategic outcomes. Journal of Business \& Industrial Marketing, 26 (6): 395-406.

Klemperer, Paul., 1995. Competition When Consumer Have Switching Costs: An Overview With Applications to Industrial Organization. Macro-economics, and International Trade. Review of Economic Studies, 62: 515-539.

Liu, C., Guo, Y. M. \& Lee, C., 2011. The effects of relationship quality and switching barriers on customer loyalty. International Journal of Information Management, 31 (1): 71-79.

Nguyễn Đình Thọ và Nguyễn Thị Mai Trang, 2011. Nghiên cứu khoa học marketing: Úng dụng mô hình cấu trúc tuyến tính SEM, ấn bản lần 2, NXB Lao Động.

Sheth, Jagdish N. \& Parvatiyar,A., 1995. Relationship Marketing in Consumer Markets: Antecedents and Consequences. Journal of Academy of Marketing Science, 23 (Fall): 255-272.

Willys, N. (2018) Customer Satisfaction, Switching Costs and Customer Loyalty: An Empirical Study on the Mobile Telecommunication Service. American Journal of Industrial and Business Management, 8, 1022-1037.

Yoo, B.H, Donthu, N \& Lee S.H., 2000. An Examination of Selected Marketing Mix Elements and Brand Equity, Journal of the Academy of Marketing Science, 28 (2), 195-211. 